\title{
Die magnetostriktive Verschiebung der ferromagnetischen Resonanz an Ni-Fe-Drähten
}

\author{
Von V. ZEHLER * \\ Aus dem Institut für theoretische Physik der Justus-Liebig-Hochschule Gießen \\ (Z. Naturforschg. 12 a, 441-442 [1957]; eingegangen am 22. Dezember 1956)
}

\begin{abstract}
Durch das Anlegen von Zugspannungen während der Untersuchung der ferromagnetischen Resonanz an Drähten wird das Resonanzmagnetfeld zu kleineren Werten hin verschoben. Es wird gezeigt, daß diese Verschiebung auf der Magnetostriktion beruht. Die quantitative Übereinstimmung zwischen Theorie und Experiment weist den Weg zu einer neuen Meßmethode der Sättigungsmagnetostriktion.
\end{abstract}

In der vorhergehenden Arbeit ${ }^{1}$ wurde die Behauptung aufgestellt, daß Zugspannungen auf Grund der Magnetostriktion die Resonanzfeldstärke zu kleineren Werten hin verschöben. Um dies zu beweisen, wurden Messungen an $\mathrm{Ni}-\mathrm{Fe}$-Drähten gemacht, die gleichzeitig mit einer Zugspannung belastet wurden. Dazu wurde eine Legierung mit $60,2 \% \mathrm{Ni}$ ausgewählt, weil hier die Magnetostriktion nahezu isotrop ist $^{2}$. Die Messung wurde an einem geglühten und elektropolierten Draht ${ }^{1}$ von etwa $0,32 \mathrm{~mm}$ Durchmesser in einem HR vom Typ TEM 004 vorgenommen $^{3}$. Die Abb. 1 zeigt die Dispersionskurve, die Resonanz liegt bei einer Feldstärke von $5,16 \cdot 10^{4}$ Amp $/ \mathrm{m}$ (entsprechend 648 Oe.). Bei dieser Messung wurde der Draht, um ein Durchhängen zu vermei- den, mit einer Zugkraft von 152 pond (entsprechend einer Zugspannung von $1,82 \cdot 10^{7} \mathrm{Newton} / \mathrm{m}^{2}$ ) belastet. Bei weiteren Versuchen wurde diese Kraft bis auf $2 \mathrm{kp}$ (entsprechend $2,4 \cdot 10^{8} \mathrm{Newton} / \mathrm{m}^{2} \approx 24 \mathrm{kp} /$ $\mathrm{mm}^{2}$ ) erhöht. Das Ergebnis der Messungen mit steigenden Zugspannungen zeigt die Abb. 2. Zwischen den einzelnen Messungen unter Zug wurden jeweils solche mit nur $152 \mathrm{p}$ Zugkraft durchgeführt, um festzustellen, ob in jedem Falle der Ausgangszustand wieder erreicht wurde. Das war stets innerhalb der Meßgenauigkeit der Fall mit Ausnahme der Messung im Anschluß an den Versuch mit $2 \mathrm{kp}$. Daher wurde die Meßreihe hier abgebrochen. Den Vergleich zweier solcher Probemessungen mit der Kurve der Abb. 1 zeigt die Abb. 3.

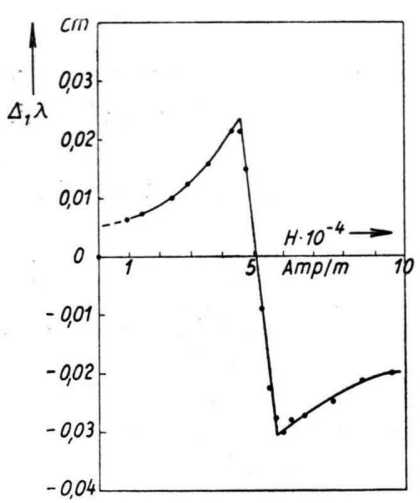

Abb. 1. Die Verschiebung der Resonanzwellenlänge $\Delta_{1} \lambda$ des $\mathrm{HR}$ (Dispersionskurve) in Abhängigkeit vom Magnetfeld $H$ für einen Ni-Fe-Draht $(60,2 \% \mathrm{Ni})$. Bei sehr kleinen Magnetfeldern ergeben sich Abweichungen, weil der Draht hier noch nicht bis zur Sättigung magnetisiert ist.

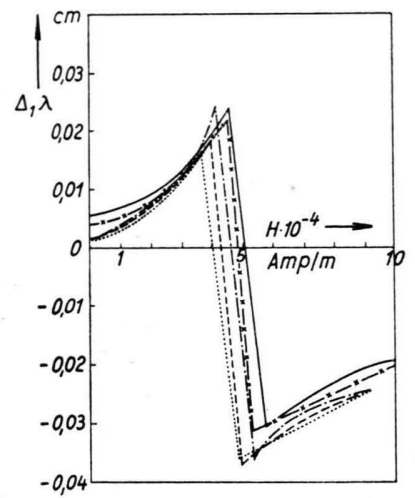

Abb. 2. Dispersionskurven des gleichen Drahtes wie in Abb. 1, bei gleichzeitiger Zugbelastung $\left(\sigma\right.$ in $10^{7}$ Newton $\left./ \mathrm{m}^{2}\right)$.

- $\sigma=1,8,-\mathrm{x}-\mathrm{x}-: \sigma=6,0$,

$-\cdot-\cdot-: \sigma=12,----: \sigma=18$, $\cdots \cdots: \sigma=24$.

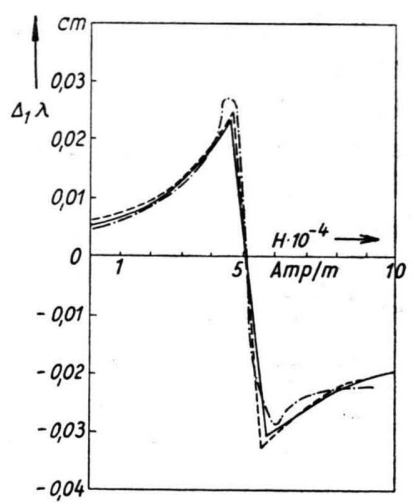

Abb. 3. Vergleich dreier Proben auf Reversibilität nach dem Anlegen von Zugspannungen. —: Bei Beginn der Meßreihe, - - - : Nach Belastung mit $18 \cdot 10^{7}$ Newton $/ \mathrm{m}^{2}$, -.-.-.- : Nach Belastung mit $24 \cdot 10^{7}$ Newton $/ \mathrm{m}^{2}$.
* Jetzt AEG-Forschungsinstitut, Frankfurt (Main)-Hausen.

1 V. Zehler, Z. Naturforschg. 12 a, 437 [1957]; im folgenden als II zitiert.
2 R. M. Bozorth, Ferromagnetism, Toronto, New York, London 1951, S. 649.

3 V. Zehler, Z. Naturforschg. 12 a, 433 [1957]; im folgenden als I zitiert. 


\section{Theoretische Deutung}

Es wurde schon in II vermutet, daß Zugspannungen wegen der Magnetostriktion wie ein zusätzliches äußeres Magnetfeld wirken. Das ist leicht folgendermaßen einzusehen: Bekanntlich übt ein mechanischer Zug auf die Spins eines ferromagnetischen Körpers ein Drehmoment aus. Dessen freie Energie hängt $a b$ von dem Winkel $\alpha$ zwischen den Spins bzw. der Magnetisierung und der Spannung $\sigma$ und lautet ${ }^{4}$

$$
E=\frac{3}{2} \lambda_{\mathrm{s}} \sigma \sin ^{2} \alpha
$$

Dabei ist $\lambda_{\mathrm{s}}$ die Sättigungsmagnetostriktion. Das Drehmoment ergibt sich durch Differentiation nach $\alpha$. Wir wollen es formal dem Drehmoment eines Ersatzmagnetfeldes $\mathfrak{H}_{\sigma}$ gleichsetzen:

$$
\begin{gathered}
\frac{\partial E}{\partial \alpha}=3 \lambda_{\mathrm{s}} \cdot \sigma \cdot \sin \alpha \cdot \cos \alpha=\left|\left[\mathfrak{M} \mathcal{T} \mathfrak{S}_{\sigma}\right]\right|, \\
3 \lambda_{\mathrm{s}} \sigma \cos \alpha=M H_{\sigma} .
\end{gathered}
$$

In unserem Falle liegen Zugspannung und Magnetisierung parallel, daher ergibt sich für das Ersatzmagnetfeld:

$$
H_{\sigma}=\frac{3 \lambda_{\mathrm{s}} \sigma}{M} .
$$

Um diesen Wert sollte sich die Resonanzfeldstärke beim Anlegen der Zugspannung $\sigma$ verschieben.

Da ein linearer Zusammenhang zwischen $\sigma$ und $H_{\sigma}$ besteht, wurde in der nächsten Abbildung die Resonanzfeldstärke in Abhängigkeit von der Zugspannung $\sigma$ aufgetragen. Die Meßpunkte liegen einer Geraden erfreulich nahe. Die Gleichung dieser Geraden wurde nach der Methode der kleinsten Quadrate approximiert. Danach ergibt sich für den Anstieg ein Wert von

$$
\begin{aligned}
\frac{3 \lambda_{\mathrm{s}}}{M}=-4,05 \cdot 10^{-5}\left[\frac{\mathrm{Amp}}{\mathrm{m}} / \frac{\text { Newton }}{\mathrm{m}^{2}}\right] \\
=-4,05 \cdot 10^{-5}\left[\frac{\mathrm{m}^{2}}{\text { Weber }}\right] .
\end{aligned}
$$

Das liefert mit einer Sättigungsmagnetisierung von $1,45 \mathrm{Weber} / \mathrm{m}^{2}$ (entsprechend $1,45 \cdot 10^{4} \mathrm{Gauß}{ }^{5}$ ) für die Sättigungsmagnetostriktion den Wert

$$
\lambda_{\mathrm{s}}=2,0 \cdot 10^{-5}
$$

in überraschend guter Übereinstimmung mit dem von Lichtenberger ${ }^{6}$ angegebenen Wert. Damit ist quantitativ nachgewiesen, daß eine Zugspannung in derselben Weise wie ein angelegtes Gleichmagnetfeld wirkt und die Resonanz verschiebt. Die Form der Dispersionskurve und damit die Dämpfung wird kaum beeinflußt, solange die Zugspannung klein genug ist, keine irreversiblen Veränderungen im Gefüge des Drahtes hervorzurufen.

Gleichzeitig zeigt sich hier ein neues Verfahren auf, durch Verfeinerung der Meßmethodik die Magnetostriktion zu messen: Die sonst übliche Unsicherheit in der Kenntnis, wie die Orientierungen der einzelnen Bereiche im unmagnetisierten Zustand verteilt sind, entfällt hier, da nur Messungen an magnetisierten Proben gemacht werden.

Der Ordinatenabschnitt der Geraden in Abb. 4 liefert das Resonanzmagnetfeld für $\sigma=0 \mathrm{mit}$ $5,22 \cdot 10^{4} \mathrm{Amp} / \mathrm{m}$; daraus folgt mit $M=1,45 \mathrm{~Wb} / \mathrm{m}^{2}$ für $\gamma=2,32 \cdot 10^{5} \mathrm{~m} /$ Amp sec bzw. $g=2,10$.

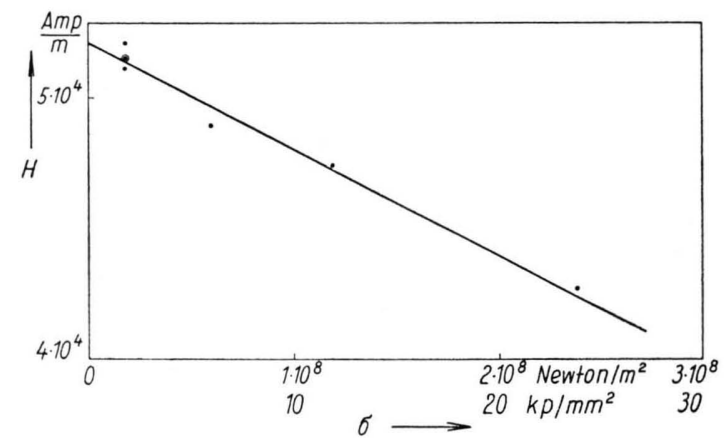

Abb. 4. Resonanzfeldstärken in Abhängigkeit von der Zug. spannung $\sigma$.

Der Deutschen Forschungsgemeinschaft danken wir, daß sie uns die Durchführung dieser Untersuchungen durch Bereitstellung von Apparaten und Mitteln ermöglicht hat. Der V a c u u m s h m e l z e A.G. Hanau danken wir für die Überlassung von Drähten. Herrn Prof. Döring für Diskussionen und Anregungen zu danken, ist mir ein besonderes Anliegen.

${ }^{4}$ R. Becker u. W. Döring, Ferromagnetismus, Berlin 1939, S. 131.

5 l. c. ${ }^{2}$, S. 109.

${ }^{6}$ F. Lichtenberger, Ann. Phys., Lpz. (5) 15. 45 [1932]. 